\title{
Images from the Haematologica Atlas of Hematologic Cytology: dysgranulopoiesis
}

\section{Rosangela Invernizzi}

University of Pavia, Pavia, Italy

E-mail: ROSANGELA INVERNIZZI - rosangela.invernizzi@unipv.it

doi:10.3324/haematol.2021.278517

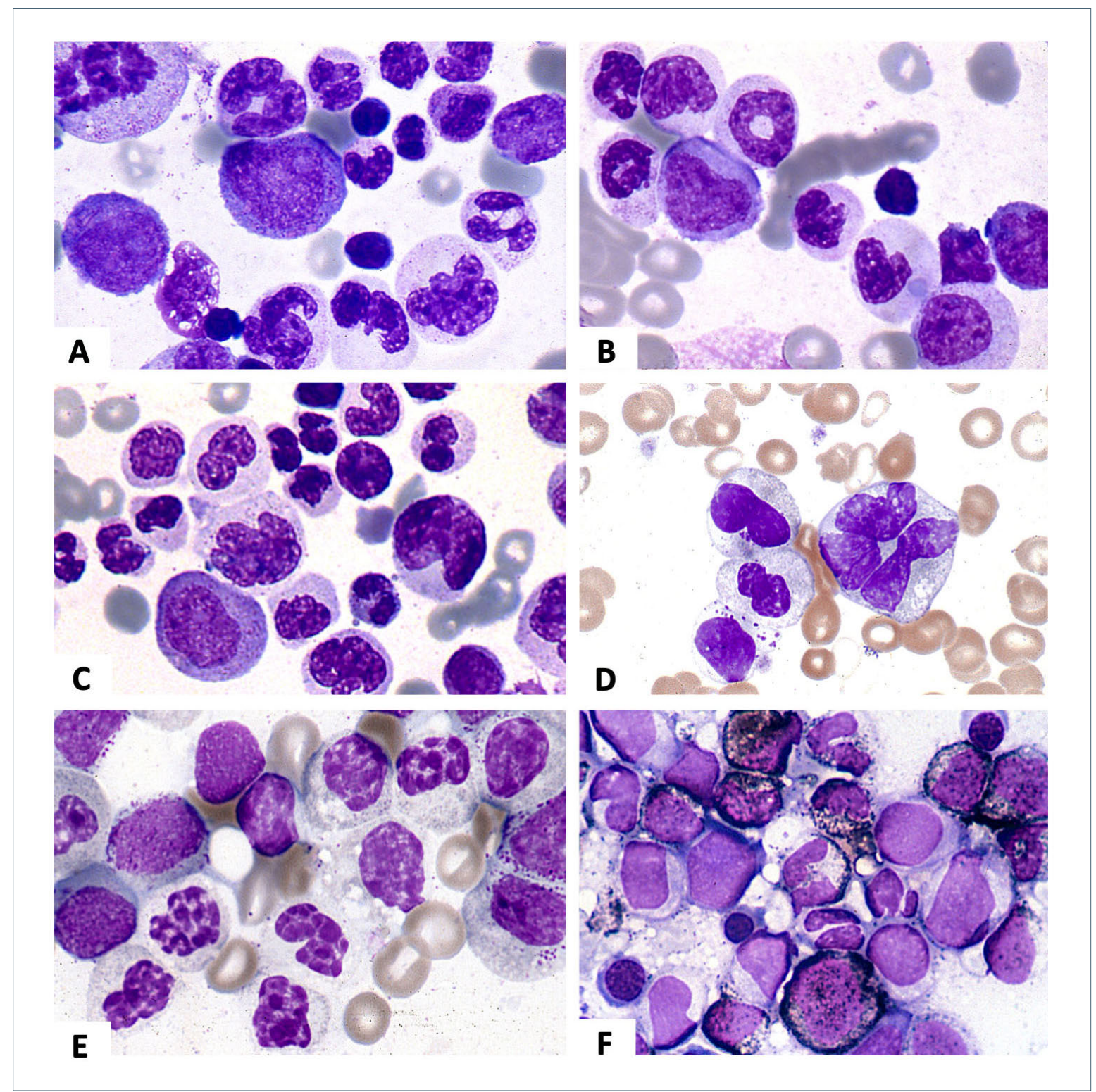

n myelodysplastic syndrome without excess blasts, the precise recognition and quantification of dysplasia is critical
for the diagnosis. The morphological abnormalities of the granulocytic series to be taken into account are illustrated
in the Figure, showing bone marrow smears. Cytoplasmic hypogranularity or agranularity is considered a highly spe-
cific dysplastic feature (Figure A-E), whereas the most frequent nuclear abnormality is hyposegmentation (pseudo-Pelger-
Huët anomaly), which is almost pathognomonic of myelodysplasia (Figure C and E). Other features of dysgranulopoiesis
include anisocytosis of neutrophils (Figure A and C), giant nuclear segments or bizarre nuclear shapes (Figure A-C),
macropolycytes (Figure D), and abnormal chromatin clumping (Figure E). Nuclear and cytoplasmic anomalies are often
associated. Hypogranularity or agranularity of neutrophils can be better assessed with Sudan black staining (Figure F) or
through the peroxidase reaction.

\section{Reference}

1. Invernizzi R. Myelodysplastic syndromes. Haematologica. 2020;105(Suppl 1):78-97. 